\title{
A Pathwise Fractional One Compartment Intra-Veinous Bolus Model
}

\author{
Nicolas Marie ${ }^{1}$ \\ ${ }^{1}$ Laboratoire Modal'X, Université Paris 10, Nanterre, France \\ Correspondence: Nicolas Marie, Laboratoire Modal'X, Université Paris 10, Nanterre, France. Tel: 33-7-7001- \\ 8351. E-mail: nmarie@u-paris10.fr
}

Received: March 24, 2014 Accepted: June 17, 2014 Online Published: June 26, 2014

doi:10.5539/ijsp.v3n3p65 URL: http://dx.doi.org/10.5539/ijsp.v3n3p65

\begin{abstract}
To extend the deterministic compartments pharmacokinetics models as diffusions seems not realistic on the biological side because the paths of these stochastic processes are not smooth enough. In order to extend the one compartment intra-veinous bolus models, this paper suggests to model the concentration process $C$ by a class of stochastic differential equations driven by a fractional Brownian motion of Hurst parameter belonging to ]1/2, 1[.

The first part of the paper provides probabilistic and statistical results on the concentration process $C$ : the distribution of $C$, a control of the uniform distance between $C$ and the solution of the associated ordinary differential equation, and consistent estimators of the elimination constant, of the Hurst parameter of the driving signal, and of the volatility constant.

The second part of the paper provides applications of these theoretical results on simulated concentrations: a method to choose the parameters on small sets of observations, and simulations of the estimators of the elimination constant and of the Hurst parameter of the driving signal. The relationship between the quality of the estimations and the size/length of the sample is discussed.
\end{abstract}

Keywords: pharmacokinetics, one compartment bolus models, fractional Brownian motion, fractional OrnsteinUhlenbeck process, ergodicity, least-square estimation

\section{Introduction}

The compartments pharmacokinetic models describe how an administered drug is transmitted among the body's compartments. The concentration of the drug in each compartment can be modeled by ordinary differential equations (see Jacomet, 1989).

In particular, in the one compartment models, the concentration is classically modeled by a linear (deterministic) differential equation with a negative constant coefficient, taking in account the absorption and the elimination steps. Only the one compartment models are studied in the current paper.

By D’Argenio and Park (1997), the elimination process has a deterministic component and a random component. A natural way to take in account these components is to add a stochastic noise in the linear differential equation which classically models the concentration. It has been studied in the Itô stochastic calculus framework by many authors (see Sen \& Bell, 2006; Donnet \& Samson, 2013).

However, as mentioned in Delattre and Lavielle (2011), since the standard Brownian motion has $\alpha$-Hölder continuous paths with $\alpha \in] 0,1 / 2$ [, the extension of the deterministic model as a diffusion is not realistic on the biological side. Delattre and Lavielle force the regularity of the paths of the concentration process $C$ by putting

$$
C_{t}:=C_{0} \exp \left(-\int_{0}^{t} D_{s} d s\right) ; t \in \mathbb{R}_{+}
$$

where $D$ is the diffusion which extends the deterministic model.

As mentioned in Marie (2014), another way to increase the regularity of the paths of the concentration process is to replace the standard Brownian motion by a fractional Brownian motion $B^{H}$ of Hurst parameter $\left.H \in\right] 1 / 2,1[$ as 
as driving signal. Since the signal is not a semi-martingale anymore, the stochastic integral is taken pathwise, in the sense of Young (see Lejay, 2010). The Young integral keeps the regularity of the driving signal, therefore the concentration process has $\alpha$-Hölder continuous paths with $\alpha \in] 0, H[$.

In both Itô and pathwise stochastic calculus frameworks, an interesting volatility function is $x \in \mathbb{R}_{+} \mapsto \sigma x^{\beta}$ with $\sigma \in \mathbb{R}$ and $\beta \in[0,1]$. It covers classical models:

- $\beta=0, \sigma \neq 0$ : Langevin equation. Its solution is the so-called Ornstein-Uhlenbeck process.

- $\beta=1 / 2, \sigma \neq 0$ : Cox-Ingersoll-Ross model.

- $\beta=1, \sigma \neq 0$ : Linear stochastic differential equation.

- $\sigma=0$ : Linear ordinary differential equation.

In the Itô stochastic calculus framework, that concentration model has been studied on the statistical side in Kalogeropoulos et al. (2008). In the pathwise stochastic calculus framework, it has been studied on probabilistic side in Marie (2014).

This paper is devoted to the probabilistic and statistical study of the special case of the one compartment intraveinous (i.v.) bolus model with a fractional Brownian signal:

$$
C_{t}=C_{0}-v \int_{0}^{t} C_{s} d s+\sigma \int_{0}^{t} C_{s}^{\beta} d B_{s}^{H} ; \quad t \in\left[0, \tau_{0}\right]
$$

where

$$
\tau_{0}:=\inf \left\{t \in \mathbb{R}_{+}: C_{t}=0\right\},
$$

the exponent $\beta$ belongs to $[0,1[, v>0$ is the rate of elimination describing the removal of the drug by all elimination processes including excretion and metabolism, and $C_{0}:=A_{0} / V$ with $A_{0}>0$ the administered dose and $V>0$ the volume of the elimination compartment.

Since its vector field is of class $C^{\infty}$ on the bounded sets of $\mathbb{R}_{+}^{*}$, Equation (1) admits a unique continuous pathwise solution defined on $\left[0, \tau_{0}\right]$ and satisfying $C .=X^{\gamma+1}$, where $\gamma:=\beta /(1-\beta)$ and $X$ is the solution of the following fractional Langevin equation:

$$
X_{t}=C_{0}^{1-\beta}-v(1-\beta) \int_{0}^{t} X_{s} d s+\sigma(1-\beta) B_{t}^{H} ; t \in \mathbb{R}_{+}
$$

That equation is obtained by applying the rough change of variable formula to the process $C$ and to the map $x \in \mathbb{R}_{+} \mapsto x^{1-\beta}$ on $\left[0, \tau_{0}\right]$. For details, the reader can refer to Marie (2014). The fractional Langevin equation is deeply studied in Cheridito et al. (2003).

Since the concentration process has to be positive and to end when it hits zero, it can be defined as the solution of Equation (1) on $\left[0, \tau_{0}\right]$.

For the sake of simplicity, even if the following equality is only true on $\left[0, \tau_{0}\right]$, throughout this paper, $C$ is defined on $\mathbb{R}_{+}$by

$$
C_{t}:=\left|C_{0}^{1-\beta}+\sigma B_{t}^{H}(\vartheta)\right|^{\gamma+1} e^{-v t} ; \quad t \in \mathbb{R}_{+}
$$

with

$$
\vartheta_{t}:=(1-\beta) e^{v(1-\beta) t} ; \quad t \in \mathbb{R}_{+}
$$

and the Young/Wiener integral (see Appendix A)

$$
B_{t}^{H}(\vartheta):=\int_{0}^{t} \vartheta_{s} d B_{s}^{H} ; \quad t \in \mathbb{R}_{+} .
$$

Note that for $H=1$ and $\beta=0$, the fractional Brownian motion coincides with $t \in \mathbb{R}_{+} \mapsto \xi t$ such that $\xi \rightsquigarrow \mathcal{N}(0,1)$, and

$$
C_{t}=\left|\frac{\sigma}{v} \xi+\left(C_{0}-\frac{\sigma}{v} \xi\right) e^{-v t}\right| ; \quad t \in \mathbb{R}_{+} .
$$

That limit case illustrates that the Hurst parameter $H$ is continuously controlling the regularity of the paths of the concentration process, but also that $\sigma$ and $H$ provide two complementary ways to control the impact of the random component on the elimination process with respect to its deterministic component. 
In mathematical finance, the semi-martingale property of the prices process is crucial in order to ensure the completeness of the market. The Itô stochastic calculus is then tailor-made to model prices in finance. In pharmacokinetics, the semi-martingale property of the concentration process seems not crucial on the biological side.

To replace the standard Brownian motion by a fractional Brownian motion in the pathwise stochastic calculus framework implies that the concentration process does not satisfy the Markov property anymore. In general, it makes the estimation of the parameters $v, \sigma$ and $H$ difficult, but the relationship between $C$ and $X$ mentioned above allows to bypass these difficulties by using results coming from $\mathrm{Hu}$ and Nualart (2010), Istas and Lang (1997), and Brouste and Iacus (2013). On the estimation of $v$ (resp. $\sigma$ and $H$ ) in a wider class of models, see also Neuenkirch and Tindel (2014) (resp. Berzin \& León, 2008).

Since the stochastic process $C$ could model the elimination process more realistically than the deterministic models, it could be used for potentially toxic drugs involving in clinical studies. For instance, the elimination of the ketamine, which can be neurotoxic but more effective than classical antidepressants in the treatment of major depressive disorders (see Correll \& Futter, 2006), could be modeled by the stochastic process studied in the current paper. On a pharmacokinetic/pharmacodynamic model of the elimination of the ketamine, see Dahan et al. (2011).

The pathwise models as Equation (1) are well adapted to population pharmacokinetics. Indeed, the pathwise stochastic calculus framework is tailor-maid to assume that the parameters of the studied equation are random.

These problems will be studied in forthcoming papers.

The second section is devoted to probabilistic and statistical properties of the processes $X$ and $C$. The first part deals with the distribution of the concentration process $C$ and a control, in probability, of the uniform distance between the fractional Ornstein-Uhlenbeck process $X$ and the solution of the associated ordinary differential equation. The second part provides a strongly consistent estimator of the elimination constant $v$, and an extension of existing ergodic theorems for the fractional Ornstein-Uhlenbeck process $X$ is established. The third part provides a strongly consistent estimator of $(H, \sigma)$. A weakly consistent estimator of $v$ is deduced when the values of $H$ and $\sigma$ are unknown.

The third section is devoted to the application of the theoretical results of the second subsection on simulated concentrations. For small sets of observations, the first part provides a method to choose the parameters $H, \sigma$ and $\beta$. The cornerstone of the method is the control of the uniform distance between $X$ and the solution of the associated ordinary differential equation mentioned above. The second part illustrates the convergence of the estimators provided at Section 2. The relationship between the quality of the estimations and the size/length of the sample is discussed.

Appendices A and B provide respectively useful definitions and results on the fractional Brownian motion, and the proofs of the results stated at Section 2.

\section{Probabilistic and Statistical Properties of the Concentration Process}

The first subsection deals with the distribution of the concentration process $C$ (see Lemma 1 and Proposition 2) and a control, in probability, of the uniform distance between the fractional Ornstein-Uhlenbeck process $X$ and the solution of the associated ordinary differential equation (see Proposition 3).

The second subsection provides a strongly consistent estimator of the elimination constant $v$ studied in Hu and Nualart (2010), and an extension of existing ergodic theorems for the fractional Ornstein-Uhlenbeck process $X$ is established.

The third subsection provides a special case of the strongly consistent estimator of $(H, \sigma)$ studied in Istas and Lang (1997) and Brouste and Iacus (2013). A weakly consistent estimator of $v$ is deduced when the values of $H$ and $\sigma$ are unknown.

Refer to Appendix B for the proofs of the results stated in this section.

\subsection{Distribution of the Concentration Process and Related Topics}

The following lemma provides a suitable expression of the covariance function of the fractional Ornstein-Uhlenbeck process $X$.

Lemma $1 B^{H}(\vartheta)$ is a centered Gaussian process of covariance function $R_{H, \vartheta}$ such that:

$$
R_{H, \vartheta}(s, t)=\alpha_{H}(1-\beta)^{2} \int_{0}^{s} \int_{0}^{t}|u-v|^{2(H-1)} e^{v(1-\beta)(u+v)} d u d v
$$


for every $s, t \in \mathbb{R}_{+}$. Then, the covariance function $R_{X}$ of the fractional Ornstein-Uhlenbeck process $X$ satisfies:

$$
R_{X}(s, t)=\alpha_{H} \sigma^{2}(1-\beta)^{2} \int_{0}^{s} \int_{0}^{t}|u-v|^{2(H-1)} e^{-v(1-\beta)[(t-u)+(s-v)]} d u d v
$$

for every $s, t \in \mathbb{R}_{+}$.

Proposition 2 For every $n \in \mathbb{N}^{*}$ and $t_{1}, \ldots, t_{n} \in \mathbb{R}_{+}$, the distribution of the random vector $\left(C_{t_{1}}, \ldots, C_{t_{n}}\right)$ has a density $\chi_{n}$ with respect to the Lebesgue measure on $\left(\mathbb{R}^{n}, \mathcal{B}\left(\mathbb{R}^{n}\right)\right)$ such that:

$$
\begin{aligned}
\chi_{n}\left(x_{1}, \ldots, x_{n}\right)= & \frac{2^{n}(1-\beta)^{n} \mathbf{1}_{\mathbb{R}_{+}^{n}}\left(x_{1}, \ldots, x_{n}\right)}{(2 \pi)^{n / 2} \sqrt{\left|\operatorname{det}\left(R_{n}\right)\right|}} \prod_{i=1}^{n} x_{i}^{-\beta} \times \\
& \exp \left[-\frac{1}{2}\left[R_{n}^{-1}\left[\left(\begin{array}{c}
x_{1}^{1-\beta} \\
\vdots \\
x_{n}^{1-\beta}
\end{array}\right)-V_{n}\right]\right] \cdot\left[\left(\begin{array}{c}
x_{1}^{1-\beta} \\
\vdots \\
x_{n}^{1-\beta}
\end{array}\right)-V_{n}\right]\right] ;\left(x_{1}, \ldots, x_{n}\right) \in \mathbb{R}^{n}
\end{aligned}
$$

where, $R_{n} \in S_{n}\left(\mathbb{R}_{+}\right)$and $V_{n} \in \mathbb{R}^{n}$ are defined by

$$
R_{n}(i, j):=\alpha_{H} \sigma^{2}(1-\beta)^{2} \int_{0}^{t_{i}} \int_{0}^{t_{j}}|u-v|^{2(H-1)} e^{-v(1-\beta)\left[\left(t_{j}-u\right)+\left(t_{i}-v\right)\right]} d u d v
$$

and

$$
V_{n}(i):=C_{0}^{1-\beta} e^{-v(1-\beta) t_{i}}
$$

for every $i, j \in\{1, \ldots, n\}$.

It is a straightforward application of Lemma 1, the equality $C .=|X .|^{\gamma+1}$, and Marie (2014), Proposition 5.1.

Let $X^{\text {det }}$ be the solution of the ordinary differential equation associated to Equation (2):

$$
X_{t}^{\text {det }}=X_{0}-v(1-\beta) \int_{0}^{t} X_{s}^{\text {det }} d s ; \quad t \in \mathbb{R}_{+} .
$$

Proposition 3 For every $x>0$ and $T>0$,

$$
\mathbb{P}\left(\left\|X-X^{\operatorname{det}}\right\|_{\infty, T}>x\right) \leqslant 2 \exp \left[-\frac{x^{2}}{2 \sigma^{2} R_{H, \vartheta}(T, T)}\right] .
$$

Let $\lambda \in] 0,1\left[\right.$ be arbitrarily chosen. By Proposition 3 , it is sufficient to assume that $\sigma^{2} \in[0, M(\lambda, x, H)]$ with

$$
M(\lambda, x, H):=\frac{x^{2}}{2 R_{H, \vartheta}(T, T) \log (2 / \lambda)},
$$

to ensure with probability greater than $1-\lambda$ that $\left|X_{t}-X_{t}^{\mathrm{det}}\right| \leqslant x \in \mathbb{R}_{+}^{*}$ for every $t \in[0, T]$.

Consider the function $C^{\mathrm{det}}: \mathbb{R}_{+} \rightarrow \mathbb{R}_{+}^{*}$ defined by $C_{t}^{\mathrm{det}}:=\left|X_{t}^{\mathrm{det}}\right|^{\gamma+1}$ for every $t \in \mathbb{R}_{+}$.

Corollary 4 For every $x>0$ and $T>0$ such that $C_{T}^{\text {det }}>x^{\gamma+1}$,

$$
\mathbb{P}\left[\forall t \in[0, T], C_{t} \in\left[\left|\left(C_{t}^{\operatorname{det}}\right)^{1-\beta}-x\right|^{\gamma+1},\left|\left(C_{t}^{\operatorname{det}}\right)^{1-\beta}+x\right|^{\gamma+1}\right]\right] \geqslant 1-2 \exp \left[-\frac{x^{2}}{2 \sigma^{2} R_{H, \vartheta}(T, T)}\right] .
$$

An application of Proposition 3 and Corollary 4 is provided at Subsection 3.1.

\subsection{Ergodic Theorem and Estimator of the Elimination Constant}

Let $Y$ be the stochastic process defined by:

$$
Y_{t}:=\sigma(1-\beta) \int_{-\infty}^{t} e^{-v(1-\beta)(t-s)} d B_{s}^{H} ; \quad t \in \mathbb{R}_{+} .
$$


Theorem 5 Let $f: \mathbb{R} \rightarrow \mathbb{R}$ be a continuous function such that:

$$
\exists n \in \mathbb{N}^{*}, \exists\left(a_{1}, b_{1}, c_{1}\right), \ldots,\left(a_{n}, b_{n}, c_{n}\right) \in \mathbb{R}_{+}^{*} \times \mathbb{R}_{+}^{2}: \forall x, \varepsilon \in \mathbb{R},|f(x+\varepsilon)-f(x)| \leqslant \sum_{i=1}^{n} c_{i}(1+|x|)^{b_{i}}|\varepsilon|^{a_{i}} .
$$

Then,

$$
\frac{1}{T} \int_{0}^{T} f\left(X_{t}\right) d t \underset{T \rightarrow \infty}{\stackrel{\text { a.s. }}{\longrightarrow}} \mathbb{E}\left[f\left(Y_{0}\right)\right]<\infty .
$$

With the notations of Theorem 5 , put $f(x):=x^{n} ; x \in \mathbb{R}$. For every $x, \varepsilon \in \mathbb{R}$,

$$
\begin{aligned}
|f(x+\varepsilon)-f(x)| & \leqslant \sum_{i=0}^{n-1}\left(\begin{array}{l}
n \\
i
\end{array}\right)|x|^{i}|\varepsilon|^{n-i} \\
& \leqslant \sum_{i=0}^{n-1} p_{i}(x)|\varepsilon|^{a_{i}}
\end{aligned}
$$

where $a_{i}:=n-i$ and

$$
p_{i}(x):=\left(\begin{array}{c}
n \\
i
\end{array}\right)(1+|x|)^{i} ; x \in \mathbb{R}, i=0, \ldots, n-1 .
$$

Then, by Theorem 5 together with Hu and Nualart (2010), Lemma 5.1:

$$
\begin{aligned}
\lim _{T \rightarrow \infty} \frac{1}{T} \int_{0}^{T} X_{t}^{n} d t & =\mathbb{E}\left(Y_{0}^{n}\right) \mathbb{P} \text {-a.s. } \\
& =\left\{\begin{array}{lll}
\frac{n ! \sigma^{n}(1-\beta)^{n-n H} v^{-n H} H^{n / 2} \Gamma^{n / 2}(2 H)}{2^{n / 2}(n / 2) !} & \text { if } n \in 2 \mathbb{N}^{*} \\
0 & \text { if } n \in \mathbb{N}^{*}-\left(2 \mathbb{N}^{*}\right)
\end{array}\right.
\end{aligned}
$$

For $n=2$, (3) coincides with Hu and Nualart (2010), Lemma 3.3.

Assume that the values of the parameters $H$ and $\sigma$ are known. For $T>0$ arbitrarily chosen, consider

$$
\widehat{v}_{T}:=\frac{1}{1-\beta}\left[\frac{1}{\sigma^{2}(1-\beta)^{2} H \Gamma(2 H) T} \int_{0}^{T} X_{t}^{2} d t\right]^{-1 /(2 H)} .
$$

Proposition $6 \widehat{v}_{T}$ is a strongly consistent estimator of $v$.

It is a straightforward consequence of (3) for $n=2$. The estimator $\widehat{v}_{T}$ is studied in Hu and Nualart (2010), Section 4. In particular, a central limit theorem is proved for $\widehat{v}_{T}$ when $\left.H \in\right] 1 / 2,3 / 4[$ (see Hu \& Nualart, 2010, Theorem 4.1).

\subsection{Estimators of the Hurst Parameter and of the Volatility Constant}

Assume that the concentration process $C$ is discretely observed at times $t_{0}, \ldots, t_{n}$, where $n \in \mathbb{N}^{*}, t_{k}:=k \delta_{n}$ for every $k \in\{0, \ldots, n\}$, and $\left(\delta_{n}\right)_{n \in \mathbb{N}}$ is a strictly positive real sequence such that:

$$
\lim _{n \rightarrow \infty} \delta_{n}=0 \text { and } \lim _{n \rightarrow \infty} n \delta_{n}=\infty .
$$

\section{Proposition 7 Consider}

$$
\widehat{H}_{n}:=\frac{1}{2} \log _{2}\left(\frac{\sum_{k=2}^{n-2}\left|X_{t_{k+2}}-2 X_{t_{k}}+X_{t_{k-2}}\right|^{2}}{\sum_{k=1}^{n-1}\left|X_{t_{k+1}}-2 X_{t_{k}}+X_{t_{k-1}}\right|^{2}}\right)
$$

$\widehat{H}_{n}$ is a strongly consistent estimator of $H$. 
Proposition 8 Consider $a_{0}:=-1 / 4, a_{1}:=1 / 2, a_{2}:=-1 / 4$ and

$$
\widehat{\sigma}_{n}:=\frac{1}{1-\beta}\left(-\frac{1}{8} \times \frac{\sum_{k=1}^{n-1}\left|X_{t_{k+1}}-2 X_{t_{k}}+X_{t_{k-1}}\right|^{2}}{\sum_{k, l=0}^{2} a_{k} a_{l}|k-l|^{2 \widehat{H}_{n}} \delta_{n}^{2 \widehat{H}_{n}}}\right)^{1 / 2} .
$$

$\widehat{\sigma}_{n}$ is a strongly consistent estimator of $\sigma$.

Refer to Brouste and Iacus (2013), Theorem 1, based on Istas and Lang (1997), Theorem 3, for a proof of Proposition 7 and Proposition 8.

The R-package Yuima, developed by A. Brouste and S. Iacus, allows to compute an estimation of $(H, \sigma)$ via $\left(\widehat{H}_{n}, \widehat{\sigma}_{n}\right)$ from observations of the fractional Ornstein-Uhlenbeck process $X$.

Proposition 9 Consider

$$
\widehat{v}_{n}^{*}:=\frac{1}{1-\beta}\left[\frac{1}{\widehat{\sigma}_{n}^{2}(1-\beta)^{2} \widehat{H}_{n} \Gamma\left(2 \widehat{H}_{n}\right) n} \sum_{k=0}^{n-1} X_{k \delta_{n}}^{2}\right]^{-1 /\left(2 \widehat{H}_{n}\right)} .
$$

$\widehat{v}_{n}^{*}$ is a weakly consistent estimator of $v$.

\section{Numerical Simulations and Pharmacokinetics}

For small sets of observations, the first subsection provides a method to choose the parameters $H, \sigma$ and $\beta$. Proposition 3 is the cornerstone of the method. The second subsection illustrates the convergence of the estimators provided at Section 2. The relationship between the quality of the estimations and the size/length of the sample is discussed.

\subsection{A Method to Choose H, $\sigma$ and $\beta$ on Small Sets of Observations}

Consider $n \in \mathbb{N}^{*}$ and $\left(t_{1}, \ldots, t_{n}\right) \in \mathbb{R}_{+}^{n}$ satisfying $t_{1}<\cdots<t_{n} \leqslant T$. Throughout this subsection, assume that the concentrations have been observed at times $t_{1}, \ldots, t_{n}$. These concentrations $c_{1}, \ldots, c_{n}$ provide observations $x_{1}, \ldots, x_{n}$ of the fractional Ornstein-Uhlenbeck process $X$ by putting $x_{i}:=c_{i}^{1-\beta} ; i=1, \ldots, n$.

Consider the following values of the other parameters involving in Equation (1), coming from Jacomet (1989), Chapter II.3:

\begin{tabular}{cc}
\hline Parameters & Values \\
\hline$T$ & $3 \mathrm{~h}$ \\
$v$ & $3.5 \mathrm{~h}^{-1}$ \\
$C_{0}$ & $1 \mathrm{~g}$ \\
\hline$n$ & 500 \\
\hline
\end{tabular}

In order to choose $H, \sigma$ and $\beta$, a method is provided by using these values as an example.

Let $\lambda \in] 0,1[$ be arbitrarily chosen. On one hand, as mentioned at Subsection 2.1, by Proposition 3; it is sufficient to assume that $\sigma^{2} \in[0, M(\lambda, x, H)]$ with

$$
M(\lambda, x, H):=\frac{x^{2}}{2 R_{H, \vartheta}(T, T) \log (2 / \lambda)},
$$

to ensure with probability greater that $1-\lambda$ that $\left|X_{t}-X_{t}^{\mathrm{det}}\right| \leqslant x \in \mathbb{R}_{+}^{*}$ for every $t \in[0, T]$. If in addition $X_{T}^{\mathrm{det}}>x$, by Corollary 4:

$$
\mathbb{P}\left[\forall t \in[0, T], C_{t} \in\left[\left|\left(C_{t}^{\operatorname{det}}\right)^{1-\beta}-x\right|^{\gamma+1},\left|\left(C_{t}^{\operatorname{det}}\right)^{1-\beta}+x\right|^{\gamma+1}\right]\right] \geqslant 1-\lambda .
$$

On the other hand, as mentioned in the introduction, the Hölder regularity of the paths of the concentration process is controlled by the Hurst parameter $H$.

The method stated bellow is based on these two points, and allows to choose the parameters $H, \sigma$ and $\beta$ on small sets of observations.

For $H=0.9$ and $\beta=0.9$, the following array provides the values of $M(\lambda, x, H)$ for the usual levels $\lambda=$ $0.01,0.05,0.10$ : 


\begin{tabular}{cccc}
\hline$x^{\gamma+1}-\lambda$ & 0.01 & 0.05 & 0.10 \\
\hline 0.1 & 0.26 & 0.38 & 0.46 \\
0.2 & 0.30 & 0.43 & 0.53 \\
0.4 & 0.36 & 0.50 & 0.61 \\
\hline
\end{tabular}

On the two following figures, some paths of the process $C$ are respectively plotted for the extreme cases $\sigma^{2}=0.26$ and $\sigma^{2}=4>0.61$. The paths of the concentration process are plotted in black and the associated deterministic model is plotted in red:

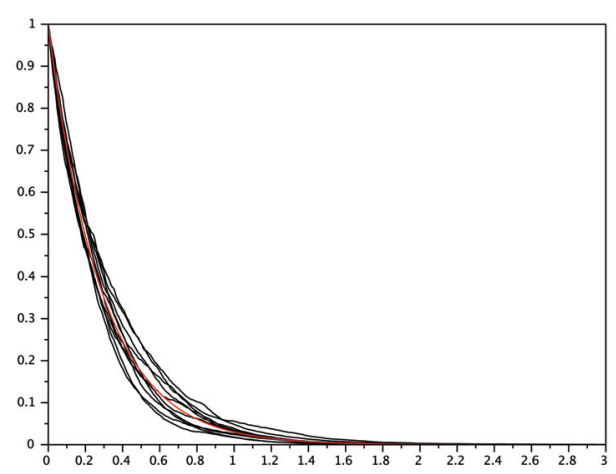

Figure 1. $(H, \sigma)=(0.9, \sqrt{0.26})$

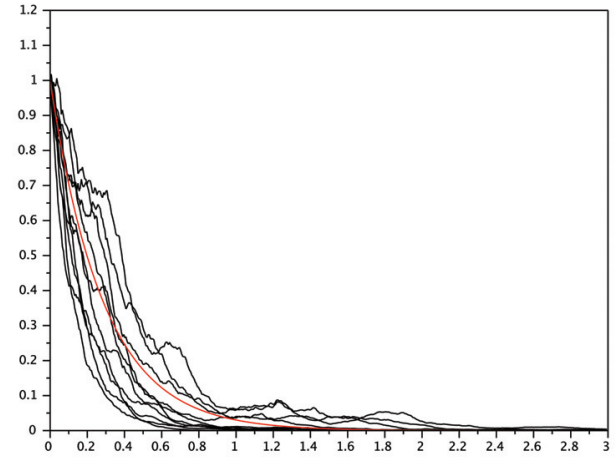

Figure 2. $(H, \sigma)=(0.9,2)$

For $H=0.6$ and $\beta=0.9$, the following array provides the values of $M(\lambda, x, H)$ for the usual levels $\lambda=$ $0.01,0.05,0.10$ :

\begin{tabular}{cccc}
\hline$x^{\gamma+1}-\lambda$ & 0.01 & 0.05 & 0.10 \\
\hline 0.1 & 0.70 & 1.00 & 1.23 \\
0.2 & 0.80 & 1.15 & 1.42 \\
0.4 & 0.92 & 1.32 & 1.63 \\
\hline
\end{tabular}

On the two following figures, some paths of the process $C$ are plotted as for $H=0.9$ :

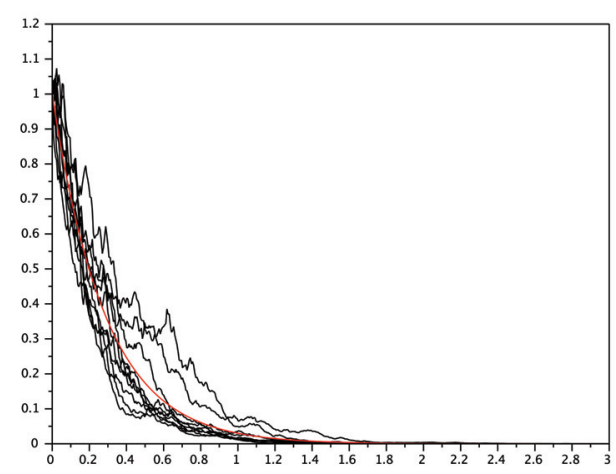

Figure 3. $(H, \sigma)=(0.6, \sqrt{0.70})$

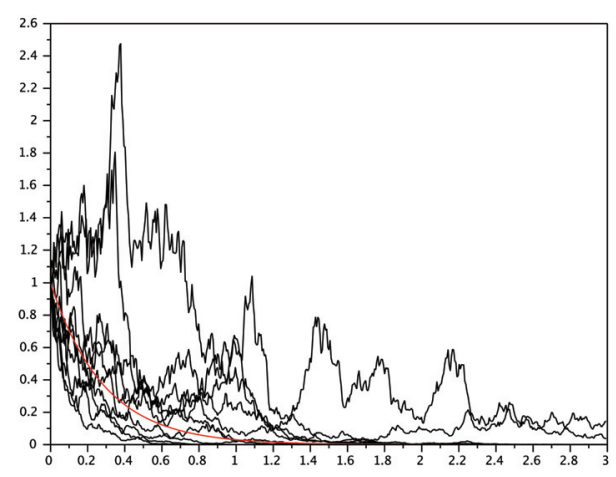

Figure 4. $(H, \sigma)=(0.6,2)$

In order to model the concentration process realistically, it is adapted to take $H=0.9$ and, for instance:

$$
\begin{aligned}
\sigma^{2} & \left.\in] 0 ; M\left(0.01,0.2^{1-\beta}, 0.9\right)\right] \\
& =] 0,0.30] .
\end{aligned}
$$

Indeed,

- For $H=0.9$ with $\sigma^{2}=4>M\left(0.10,0.4^{1-\beta}, 0.9\right)=0.61$, the paths of the concentration process seem locally regular enough, but not globally. 
- For $H=0.6$ with $\sigma^{2}=M\left(0.01,0.1^{1-\beta}, 0.6\right)=0.70$, the paths of the concentration process seem globally regular enough, but not locally.

- For $H=0.6$ with $\sigma^{2}=4>M\left(0.10,0.4^{1-\beta}, 0.6\right)=1.63$, the paths of the concentration process seem not regular enough locally and globally.

To take $\beta=0.9$ is also adapted, because if $\beta \in[0,0.8]$, for every

$$
\sigma \in\left[M\left(0.01,0.1^{1-\beta}, 0.9\right) ; M\left(0.10,0.4^{1-\beta}, 0.6\right)\right]
$$

the paths of the concentration process seem not significantly perturbed with respect to the associated deterministic model. Then, to take $\beta=0.9$ ensures that the value of the parameter $\sigma$ can be chosen such that the following realistic condition is satisfied:

$$
\mathbb{P}\left(\forall t \in[0, T], X_{t} \in\left[X_{t}^{\mathrm{det}}-0.2^{1-\beta}, X_{t}^{\mathrm{det}}+0.2^{1-\beta}\right]\right) \geqslant 0.99 .
$$

On the observed concentrations $c_{1}, \ldots, c_{n}$, the following method allows to choose $H, \sigma$ and $\beta$ :

- Step 1. Take $H \in] 0.5,1$ [ close to 1 , as 0.9 .

- Step 2. Take $\beta \in] 0,1[$.

- Step 3. Choose a standard level $\lambda \in] 0,1[$ as 0.01 or 0.05 , and put for instance

$$
\begin{aligned}
x & :=\max _{i=1}^{n}\left|c_{i}^{1-\beta}-\left(C_{0} e^{-v t_{i}}\right)^{1-\beta}\right| \\
& =\max _{i=1}^{n}\left|x_{i}-C_{0}^{1-\beta} e^{-v(1-\beta) t_{i}}\right| .
\end{aligned}
$$

Then, compute $M(\lambda, x, H)$.

If the value of $v$ is unknown, since the paths of the concentration process have to be moderately perturbed with respect to the associated deterministic model, it can be approximated by linear regression as in Jacomet (1989) (see Subsection 3.2).

- Step 4. Take $\left.\left.\sigma^{2} \in\right] 0 ; M(\lambda, x, H)\right]$ such that the paths of the concentration process are regular enough locally and globally to model the elimination of the administered drug.

If the paths of the concentration process are not significantly perturbed with respect to the associated deterministic model for some standard levels $\lambda \in] 0,1$, then go to the second step and choose a greater value of the parameter $\beta$. If the paths of the concentration process are not globally regular enough for some standard levels $\lambda \in] 0,1$, then go to the second step and choose a smaller value of the parameter $\beta$.

\subsection{Parameters Estimation}

Throughout this subsection, assume that the concentration process $C$ has been discretely observed at times $t_{0}, \ldots, t_{n}$, where $n \in \mathbb{N}^{*}, t_{k}:=k \delta_{n}$ for every $k \in\{0, \ldots, n\}$, and $\left(\delta_{n}\right)_{n \in \mathbb{N}}$ is a strictly positive real sequence such that:

$$
\lim _{n \rightarrow \infty} \delta_{n}=0 \text { and } \lim _{n \rightarrow \infty} n \delta_{n}=\infty .
$$

Consider the following values of the parameters involving in Equation (1):

\begin{tabular}{cl}
\hline Parameters & Values \\
\hline$T$ & $n \delta_{n} ; n=10, \ldots, 10^{3}$ \\
$\beta$ & 0 \\
$v$ & $1.5 \mathrm{~h}^{-1}$ \\
$H$ & 0.9 \\
$\sigma^{2}$ & 0.26 \\
$C_{0}$ & $1 \mathrm{~g}$ \\
\hline
\end{tabular}

The two following figures illustrate the convergence of the estimators $\widehat{v}_{n}$ and $\widehat{H}_{n}$ provided at Proposition 6 and Proposition 7 respectively. For every $n$ belonging to $\left\{10, \ldots, 10^{3}\right\}$, the concentration process $C$ is simulated at the 
times $t_{0}, \ldots, t_{n}$ and estimations of $v$ and $H$ are computed on these simulated observations denoted by $c_{1}, \ldots, c_{n}$ via $\widehat{v}_{n}$ and $\widehat{H}_{n}$. The estimations are plotted in black and the values of the parameters are plotted in red:

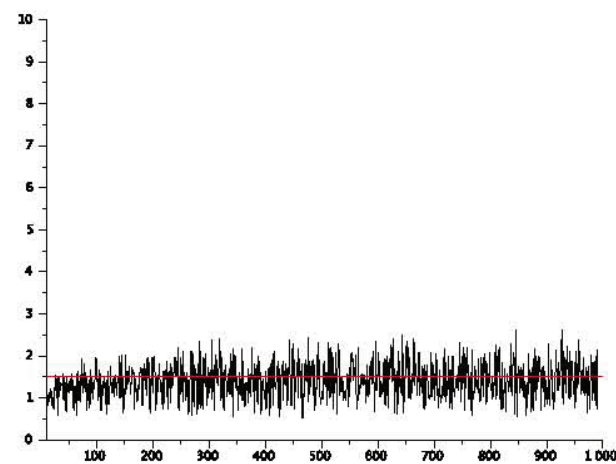

Figure $5 . \widehat{v}_{n} ; n=10, \ldots, 10^{3}$

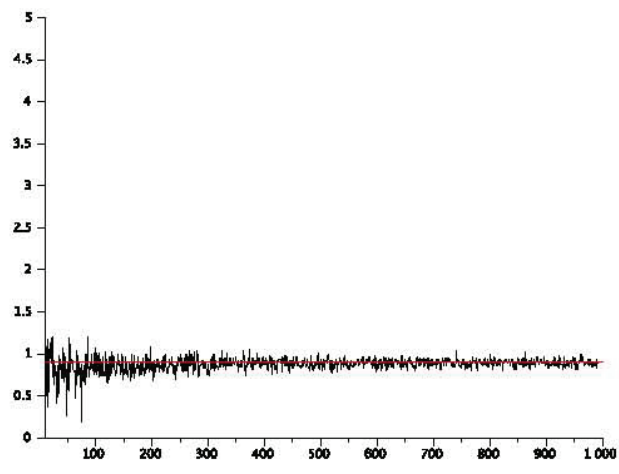

Figure 6. $\widehat{H}_{n} ; n=10, \ldots, 10^{3}$

The estimator $\widehat{v}_{n}$ converges slowly to the elimination constant $v$. Then, if the number $n$ of observations is insufficient, since the paths of the concentration process have to be moderately perturbed with respect to the associated deterministic model, it is possible de take

$$
v \approx-\frac{\operatorname{cov}\left[t_{1}, \ldots, t_{n} ; \log \left(c_{1}\right), \ldots, \log \left(c_{n}\right)\right]}{\operatorname{var}\left(t_{1}, \ldots, t_{n}\right)}
$$

as in Jacomet (1989). The method provided at Subsection 3.1 is also an alternative to choose $H$ and $\sigma$ on small sets of observed concentrations.

\section{Discussion and Perspectives}

The stochastic model studied in this paper is a natural extension of usual deterministic models used in pharmacokinetics. It has smooth enough paths to take realistically in account the random component of the elimination process, and its explicit expression together with the Decreusefond-Lavaud method allow to simulate it easily. As mentioned at Section 3, the estimators of the parameters $v, H$ and $\sigma$ provide good estimations for large sets of observed concentrations. The method described at Subsection 3.1 is well adapted to small sets of observations. For these reasons, the model could be used in clinical applications.

Assume that the therapeutic response $R_{t}$ to the administered drug at time $t \in\left[0, \tau_{0}\right]$ satisfies $R_{t}:=F\left(C_{t}, O_{t}\right)$, where $F \in C^{1}\left(\mathbb{R}^{2} ; \mathbb{R}\right)$ and $O$ is a stochastic process with $\mathbb{R}$-valued paths, not depending on the initial concentration $C_{0}=A / V$. The random variable $C_{t}$ is derivable with respect to $C_{0}>0$ and

$$
\frac{\partial R_{t}}{\partial C_{0}}=C_{0}^{-\beta}\left[C_{0}^{1-\beta}+\sigma B_{t}^{H}(\vartheta)\right]^{\gamma} e^{-v t} \partial_{1} F\left(C_{t}, O_{t}\right) .
$$

Differential calculus could then allow to compute the dose maximizing the therapeutic response $R_{t}$ for some well chosen functions $F$ and well chosen stochastic processes $O$. On the sensitivity analysis in pharmacokinetics, see Abraham et al. (2007).

Since the stochastic process $C$ seems to model the elimination process more realistically than the deterministic function $C^{\text {det }}$, the perspective of clinical applications described above could be interesting for potentially toxic drugs.

For instance, the elimination of the ketamine, that can be neurotoxic but more effective than classical antidepressants in the treatment of major depressive disorders (see Correll \& Futter, 2006), could be modeled by the stochastic process studied in the current paper. To choose $F$ and $O$ such that $R_{t}$ models the Hamilton rating scale or the Beck depression inventory at time $t$ could allow to compute the dose of ketamine maximizing its antidepressant effect and minimizing its neurotoxic effect. On a pharmacokinetic/pharmacodynamic model of the elimination of the ketamine, see Dahan et al. (2011).

Throughout the paper, the model has been studied for one patient. The population pharmacokinetics consists in making sparse measurements of the concentration of the drug on several patients. In a population model, the coefficients are random. 
The pathwise models as Equation (1) are well adapted to population pharmacokinetics. Indeed, the pathwise stochastic calculus framework is tailor-maid to assume that the parameters of the studied equation are random.

These two problems will be studied in forthcoming papers.

Finally, even if this paper deals with an application in pharmacokinetics, note that the studied model could be used in various degradation processes in physics and chemistry.

\section{Acknowledgements}

Many thanks to Alexandre Brouste for his advices about the estimation of the parameters of the fractional OrnsteinUhlenbeck process, and to Francis Lavergne M. D. for his advices about possible clinical applications of the results of this paper.

\section{References}

Abraham, A. K., Krzyzanski, W., \& Mager, D. E. (2007). Partial Derivative-Based Sensitivity Analysis of Models Describing Target-Mediated Drug Disposition. The AAPS Journal, 9(2), 181-189. http://dx.doi.org/10.1208/aapsj0902020

Adler, R. J. (1990). An Introduction to Continuity, Extrema, and Related Topics for General Gaussian Processes. Institute of Mathematical Statistics, Lecture Notes-Monograph Series 12.

Berzin, C., \& León, J. (2008). Estimation in Models Driven by Fractional Brownian Motion. Annales de l'Institut Henri Poincaré, 44(2), 191-213. http://dx.doi.org/10.1214/07-AIHP105

Brouste, A., \& Iacus, S. (2013). Parameter Estimation for the Discretely Observed Fractional Ornstein-Uhlenbeck Process and the Yuima R Package. Computational Statistics, 28(4), 1529-1547. http://dx.doi.org/10.1007/s00180-012-0365-6

Cheridito, P., Kawaguchi, H., \& Maejima, M. (2003). Fractional Ornstein-Uhlenbeck Processes. Electronic Journal of Probability, 8(3), 1-14. http://dx.doi.org/10.1214/EJP.v8-125

Correll, G. E., \& Futter, G. E. (2006). Two Case Studies of Patients with Major Depressive Disorder Given LowDose (Subanesthetic) Ketamine Infusions. Pain Medicine, 7(1), 92-95. http://dx.doi.org/10.1111/j.1526-4637.2006.00101.x

D’Argenio, D., \& Park, K. (1997). Uncertain Pharmacokinetics/Pharmacodynamics Systems: Design, Estimation $\begin{array}{lllll}\text { and Control. Control Engineering Practice, } & \text { 5(12), }\end{array}$ http://dx.doi.org/10.1016/S0967-0661(97)10025-9

Decreusefond, L., \& Lavaud, N. (1996). Simulation of the Fractional Brownian Motion and Application to the Fluid Queue. Proceedings of the ATNAC'96 conference.

Decreusefond, L., \& Ustünel, A. S. (1999). Stochastic Analysis of the Fractional Brownian Motion. Potential Analysis, 10, 177-214.

Dahan, A., Olofsen, E., Sigtermans, M., Noppers, I., Niesters, M., Aarts, L., ... Sarton, E. (2011). Population Pharmacokinetic Pharmacodynamic Modeling of Ketamine-Induced Pain Relief of Chronic Pain. European Journal of Pain, 15, 258-267.

Delattre, M., \& Lavielle, M. (2011). Pharmacokinetics and Stochastic Differential Equations: Model and Methodology. Proceedings of the Annual Meeting of the Population Approach Group in Europe.

Dieker, T. (2004). Simulation of the Fractional Brownian Motion. Master thesis, University of Twente.

Donnet, S., \& Samson, A. (2013). A Review on Estimation of Stochastic Differential Equations for Pharmacokinetic/Pharmacodynamic Models. Advanced Drug Delivery Reviews, 65(7), 929-939. http://dx.doi.org/10.1016/j.addr.2013.03.005

Hu, Y., \& Nualart, D. (2010). Parameter Estimation for Fractional Ornstein-Uhlenbeck Processes. Statistics and Probability Letters, 80, 11-12, 1030-1038. http://dx.doi.org/10.1016/j.spl.2010.02.018

Istas, J., \& Lang, G. (1997). Quadratic Variations and Estimation of the Local Hölder Index of a Gaussian Process. Annales de l'Institut Henri Poincaré, B33(4), 407-436.

Jacomet, Y. (1989). Pharmacocinétique. Tomes I et II. U.E.R. de Médecine de Nice, Ellipses. 
Kalogeropoulos, K., Demiris, N., \& Papaspiliopoulos, O. (2008). Diffusion-Driven Models for Physiological Processes. Proceedings of the IWAP conference.

Lejay, A. (2010). Controlled Differential Equations as Young Integrals: A Simple Approach. Journal of Differential Equations, 249(8), 1777-1798. http://dx.doi.org/10.1016/j.jde.2010.05.006

Marie, N. (2014). A Generalized Mean-Reverting Equation and Applications. ESAIM: PS. http://dx.doi.org/10.1051/ps/2014002

Neuenkirch, A., \& Tindel, S. (2014). A Least Square-Type Procedure for Parameter Estimation in Stochastic Differential Equations with Additive Fractional Noises. Statistical Inference for Stochastic Processes, 17(1), 99-120. http://dx.doi.org/10.1007/s11203-013-9084-z

Neveu, J. (1968). Processus aléatoires gaussiens. Presses de l’Université de Montréal.

Nualart, D. (2006). The Malliavin Calculus and Related Topics (2nd ed.). Probability and its Applications, Springer.

Sen, P., \& Bell, D. (2006). A Model for the Interaction of Two Chemicals. Journal of Theoretical Biology, 238(3), 652-656. http://dx.doi.org/10.1016/j.jtbi.2005.06.013 


\section{Appendix A. Fractional Brownian Motion}

Inspired by Nualart (2006) and, Decreusefond and Ustünel (1999), this appendix deals with the fractional Brownian motion $B^{H}$ of Hurst parameter $\left.H \in\right] 1 / 2,1$ [, its reproducing kernel Hilbert space and the Young/Wiener integral with respect to $B^{H}$. On the Gaussian processes, the reader can refer to Neveu (1968).

For a time $T>0$ arbitrarily chosen, consider

$$
\Delta_{T}:=\left\{(s, t) \in[0, T]^{2}: s<t\right\}
$$

Definition A.1 A fractional Brownian motion of Hurst parameter $H \in] 0,1]$ is a centered Gaussian process $B^{H}$ of covariance function $R_{H}$ defined by:

$$
R_{H}(s, t):=\frac{1}{2}\left(s^{2 H}+t^{2 H}-|t-s|^{2 H}\right) ; s, t \in[0, T] .
$$

The process $B^{H}$ is a semi-martingale if and only if $H=1 / 2$ (see Nualart, 2006, Proposition 5.1.1). Then, it is not possible to integrate with respect to $B^{H}$ in the sense of Itô. However, since

$$
\mathbb{E}\left(\left|B_{t}^{H}-B_{s}^{H}\right|^{2}\right)=|t-s|^{2 H}
$$

for every $s, t \in[0, T]$, the Kolmogorov continuity criterion ensures that $B^{H}$ has $\alpha$-Hölder continuous paths with $\alpha \in] 0, H[$. Therefore, for every stochastic process $X$ with $\beta$-Hölder continuous paths such that $\alpha+\beta>1, X$ can be integrated with respect to $B^{H}$ in the sense of Young. About the Young integral, which extends the well-known Riemann-Stieljès integral, the reader can refer to Lejay (2010).

In the sequel, assume that $H \in] 1 / 2,1\left[\right.$ and put $\alpha_{H}:=H(2 H-1)$. The vector space

$$
\mathcal{H}:=\left\{h \in L^{2}([0, T] ; d t): \alpha_{H} \int_{0}^{T} \int_{0}^{T}|t-s|^{2(H-1)} h(s) h(t) d s d t<\infty\right\},
$$

equipped with the scalar product $\langle., .\rangle_{\mathcal{H}}$ defined by

$$
\langle\varphi, \psi\rangle_{\mathcal{H}}:=\alpha_{H} \int_{0}^{T} \int_{0}^{T}|t-s|^{2(H-1)} \varphi(s) \psi(t) d s d t ; \varphi, \psi \in \mathcal{H},
$$

is the reproducing kernel Hilbert space of $B^{H}$.

Proposition A.2 There exists a standard Brownian motion B such that:

$$
B_{t}^{H}=\int_{0}^{t} K_{H}(t, s) d B_{s} ; \quad t \in[0, T]
$$

where

$$
K_{H}(t, s):=c_{H} s^{1 / 2-H} \int_{s}^{t}(u-s)^{H-3 / 2} u^{H-1 / 2} d u ;(s, t) \in \Delta_{T}
$$

and $c_{H}>0$ denotes a deterministic constant only depending on $H$.

$$
\mathbf{B}^{H}(h):=\int_{0}^{T}\left(K_{H}^{*} h\right)(t) d B_{t} ; h \in \mathcal{H}
$$

where

$$
\left(K_{H}^{*} h\right)(s):=\int_{s}^{T} \varphi(t) \frac{\partial K_{H}}{\partial t}(t, s) d t ; s \in[0, T]
$$

defines an iso-normal Gaussian process on $\mathcal{H}$, called Wiener integral with respect to $B^{H}$.

That proposition summarizes several results proved at Nualart (2006), Section 5.1.3.

On one hand, since the Wiener integral defined at Proposition A.2 is an iso-normal Gaussian process, it satisfies:

$$
\forall \varphi, \psi \in \mathcal{H}, \mathbb{E}\left[\mathbf{B}^{H}(\varphi) \mathbf{B}^{H}(\psi)\right]=\langle\varphi, \psi\rangle_{\mathcal{H}}
$$


On the other hand, the Hölder continuous functions on $[0, T]$ belong to $\mathcal{H}$. Then, for every (deterministic) $\beta$ Hölder continuous function $h:[0, T] \rightarrow \mathbb{R}$ such that $\alpha+\beta>1$, the Young integral of $h$ with respect to $B^{H}$ on $[0, T]$ coincides with the Wiener integral $\mathbf{B}^{H}(h)$.

There are many methods to simulate sample paths of a fractional Brownian motion. The most popular methods are the Wood-Chang algorithm (exact method) and the wavelet-based simulation (approximate method). Refer to Dieker (2004) for a survey on the simulation of the fractional Brownian motion.

This appendix concludes on the Decreusefond-Lavaud method (see Decreusefond \& Lavaud, 1996), which is easy to implement. It is based on the Volterra representation of $B^{H}$ provided at Proposition A.2. For $i=0, \ldots, n$, consider $t_{i}:=i T / n$. Then,

$$
\begin{aligned}
B_{t_{i}}^{H} & \approx \sum_{j=0}^{i-1}\left[\frac{1}{t_{j+1}-t_{j}} \int_{t_{j}}^{t_{j+1}}\left(t_{i}-t\right)^{H-1 / 2} d t\right] \Delta B_{t_{j}} \\
& =\frac{(T / n)^{H-1 / 2}}{H+1 / 2} \sum_{j=0}^{i-1}\left[(i-j)^{H+1 / 2}-(i-j-1)^{H+1 / 2}\right] \Delta B_{t_{j}}
\end{aligned}
$$

by putting $\Delta B_{t_{j}}:=(T / n)^{1 / 2} \xi_{j}$ for $j=0, \ldots, n-1$, where $\xi_{0}, \ldots, \xi_{n-1}$ are $n$ independent random variables of same distribution $\mathcal{N}(0,1)$.

\section{Appendix B. Proofs}

Proof of Lemma 1. For every $t \in \mathbb{R}_{+}$,

$$
B_{t}^{H}(\vartheta)=\mathbf{B}^{H}\left(\vartheta \mathbf{1}_{[0, t]}\right)
$$

where $\mathbf{B}^{H}$ is the Wiener integral with respect to $B^{H}$, defined at Proposition A.2. Then, $B^{H}(\vartheta)$ is a centered Gaussian process, and

$$
\begin{aligned}
R_{H, \vartheta}(s, t) & =\left\langle\vartheta \mathbf{1}_{[0, s]}, \vartheta \mathbf{1}_{[0, t]}\right\rangle_{\mathcal{H}} \\
& =\alpha_{H} \int_{0}^{s} \int_{0}^{t}|u-v|^{2(H-1)} \vartheta_{u} \vartheta_{v} d u d v
\end{aligned}
$$

for every $s, t \in \mathbb{R}_{+}$. Since

$$
X_{t}=\left[C_{0}^{1-\beta}+\sigma B_{t}^{H}(\vartheta)\right] e^{-v(1-\beta) t} ; \quad t \in \mathbb{R}_{+},
$$

the covariance function $R_{X}$ satisfies:

$$
\begin{aligned}
R_{X}(s, t) & =\sigma^{2} e^{-v(1-\beta)(s+t)} R_{H, \vartheta}(s, t) \\
& =\alpha_{H} \sigma^{2}(1-\beta)^{2} \int_{0}^{s} \int_{0}^{t}|u-v|^{2(H-1)} e^{-v(1-\beta)[(t-u)+(s-v)]} d u d v
\end{aligned}
$$

for every $s, t \in \mathbb{R}_{+}$.

Proof of Proposition 3. For every $t \in \mathbb{R}_{+}$,

$$
X_{t}-X_{t}^{\mathrm{det}}=-v(1-\beta) \int_{0}^{t}\left(X_{s}-X_{s}^{\mathrm{det}}\right) d s+\sigma(1-\beta) B_{t}^{H} .
$$

Then,

$$
X_{t}-X_{t}^{\mathrm{det}}=\sigma B_{t}^{H}(\vartheta) e^{-v(1-\beta) t} ; t \in \mathbb{R}_{+} .
$$

Let $T>0$ be arbitrarily chosen. Since $X-X^{\text {det }}$ is a centered Gaussian process and its paths are almost surely bounded on $[0, T]$, by Borell's inequality (see Adler, 1990, Theorem 2.1):

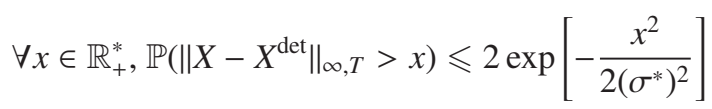

where

$$
\begin{aligned}
\left(\sigma^{*}\right)^{2} & :=\sup _{t \in[0, T]} \mathbb{E}\left(\left|X_{t}-X_{t}^{\operatorname{det}}\right|^{2}\right) \\
& =\sigma^{2} R_{H, \vartheta}(T, T) .
\end{aligned}
$$


That achieves the proof.

Proof of Corollary 4. Consider $x>0$ and $T>0$ such that $X_{T}^{\mathrm{det}}>x$. On one hand, by Proposition 3:

$$
\mathbb{P}\left(\left\|X-X^{\operatorname{det}}\right\|_{\infty, T} \leqslant x\right) \geqslant 1-2 \exp \left[-\frac{x^{2}}{2 \sigma^{2} R_{H, \vartheta}(T, T)}\right] .
$$

On the other hand, consider $\omega \in \Omega$ such that $\left\|X(\omega)-X^{\mathrm{det}}\right\|_{\infty, T} \leqslant x$. Since $X^{\mathrm{det}}$ is decreasing on $[0, T]$ :

$$
0<X_{t}^{\text {det }}-x \leqslant X_{t}(\omega) \leqslant X_{t}^{\text {det }}+x
$$

for every $t \in[0, T]$. So,

$$
\left|\left(C_{t}^{\operatorname{det}}\right)^{1-\beta}-x\right|^{\gamma+1} \leqslant C_{t}(\omega) \leqslant\left|\left(C_{t}^{\operatorname{det}}\right)^{1-\beta}+x\right|^{\gamma+1}
$$

for every $t \in[0, T]$. Therefore,

$$
\left\{\left\|X-X^{\mathrm{det}}\right\|_{\infty, T} \leqslant x\right\} \subset\left\{\forall t \in[0, T], C_{t} \in\left[\left|\left(C_{t}^{\mathrm{det}}\right)^{1-\beta}-x\right|^{\gamma+1},\left|\left(C_{t}^{\mathrm{det}}\right)^{1-\beta}+x\right|^{\gamma+1}\right]\right\} .
$$

That achieves the proof by Inequality (4).

Proof of Theorem 5. If $f$ is continuously differentiable, the ergodic theorem stated at Neuenkirch and Tindel (2011), Proposition 2.3 allows to conclude. In the special case of the fractional Ornstein-Uhlenbeck process, that additional condition is not required.

Since $Y$ is a centered, stationary and ergodic Gaussian process (see Cheridito et al., 2003), by Birkhoff-Chintchin's ergodic theorem together with Fernique's theorem:

$$
\frac{1}{T} \int_{0}^{T} f\left(Y_{t}\right) d t \underset{T \rightarrow \infty}{\stackrel{\text { a.s. }}{\longrightarrow}} \mathbb{E}\left[f\left(Y_{0}\right)\right]<\infty .
$$

Since $X_{t}=Y_{t}+\left(X_{0}-Y_{0}\right) e^{-v(1-\beta) t}$ for every $t \in \mathbb{R}_{+}$, it is sufficient to prove that:

$$
\begin{aligned}
\delta_{T}:= & \frac{1}{T}\left|\int_{0}^{T} f\left(X_{t}\right) d t-\int_{0}^{T} f\left(Y_{t}\right) d t\right| \\
\underset{T \rightarrow \infty}{\text { a.s. }} \rightarrow & 0 .
\end{aligned}
$$

Let $T>0$ be arbitrarily chosen, and put $p_{i}(x):=c_{i}(1+|x|)^{b_{i}} ; x \in \mathbb{R}, i \in\{1, \ldots, n\}$. Then,

$$
\begin{aligned}
\delta_{T} & \leqslant \frac{1}{T} \int_{0}^{T}\left|f\left[Y_{t}+\left(X_{0}-Y_{0}\right) e^{-v(1-\beta) t}\right]-f\left(Y_{t}\right)\right| d t \\
& \leqslant \frac{1}{T} \sum_{i=1}^{n}\left|X_{0}-Y_{0}\right|^{a_{i}} \int_{0}^{T} p_{i}\left(Y_{t}\right) e^{-a_{i} v(1-\beta) t} d t .
\end{aligned}
$$

For $i=1, \ldots, n$, by Cauchy-Schwarz's inequality:

$$
\left.\frac{1}{T} \int_{0}^{T} p_{i}\left(Y_{t}\right) e^{-a_{i} v(1-\beta) t} d t \leqslant \underset{\substack{\text { a.s. } \\ T \rightarrow \infty}}{[} \frac{1}{T} \int_{0}^{T} p_{i}^{2}\left(Y_{t}\right) d t\right]^{1 / 2}\left[\frac{1}{T} \int_{0}^{T} e^{-2 a_{i} v(1-\beta) t} d t\right]^{1 / 2}
$$

because

$$
\frac{1}{T} \int_{0}^{T} e^{-2 a_{i} v(1-\beta) t} d t=\frac{1}{\underset{T \rightarrow \infty}{\longrightarrow} 0}\left[e^{-2 a_{i} v(1-\beta) T}-1\right]
$$

and

$$
\frac{1}{T} \int_{0}^{T} p_{i}^{2}\left(Y_{t}\right) d t \underset{T \rightarrow \infty}{\stackrel{\text { a.s. }}{\longrightarrow}} \mathbb{E}\left[p_{i}^{2}\left(Y_{0}\right)\right]<\infty
$$


by Birkhoff-Chintchin's ergodic theorem together with Fernique's theorem. That achieves the proof.

Proof of Proposition 9. The fractional Ornstein-Uhlenbeck process $X$ satisfies:

$$
\forall p>0, \sup _{T \in \mathbb{R}_{+}} \mathbb{E}\left(\left|X_{T}\right|^{p}\right)<\infty .
$$

For every $k \in\{0, \ldots, n-1\}$ and $t \in\left[t_{k}, t_{k+1}\right]$,

$$
\begin{aligned}
\mathbb{E}^{1 / 2}\left(\left|X_{t}-X_{t_{k}}\right|^{2}\right) & \leqslant v(1-\beta) \sup _{T \in \mathbb{R}_{+}} \mathbb{E}^{1 / 2}\left(X_{T}^{2}\right)\left|t-t_{k}\right|+\sigma(1-\beta) \mathbb{E}^{1 / 2}\left(\left|B_{t}^{H}-B_{t_{k}}^{H}\right|^{2}\right) \\
& \leqslant(1-\beta)\left[v \sup _{T \in \mathbb{R}_{+}} \mathbb{E}^{1 / 2}\left(X_{T}^{2}\right)+\sigma\right]\left|t-t_{k}\right|^{H} .
\end{aligned}
$$

Then,

$$
\begin{aligned}
\mathbb{E}\left[\left|\frac{1}{n \delta_{n}} \int_{0}^{n \delta_{n}} X_{t}^{2} d t-\frac{1}{n} \sum_{k=0}^{n-1} X_{k \delta_{n}}^{2}\right|\right] & =\frac{1}{n \delta_{n}} \mathbb{E}\left[\left|\sum_{k=0}^{n-1} \int_{t_{k}}^{t_{k+1}}\left(X_{t}^{2}-X_{t_{k}}^{2}\right) d t\right|\right] \\
& \leqslant \frac{2}{n \delta_{n}} \sup _{T \in \mathbb{R}_{+}} \mathbb{E}^{1 / 2}\left(X_{T}^{2}\right) \sum_{k=0}^{n-1} \int_{t_{k}}^{t_{k+1}} \mathbb{E}^{1 / 2}\left(\left|X_{t}-X_{t_{k}}\right|^{2}\right) d t \\
& \leqslant \frac{C}{n \delta_{n}} \sum_{k=0}^{n-1} \int_{t_{k}}^{t_{k+1}}\left|t-t_{k}\right|^{H} d t=\frac{C}{H+1} \delta_{n}^{H}
\end{aligned}
$$

with

$$
C:=2(1-\beta) \sup _{T \in \mathbb{R}_{+}} \mathbb{E}^{1 / 2}\left(X_{T}^{2}\right)\left[v \sup _{T \in \mathbb{R}_{+}} \mathbb{E}^{1 / 2}\left(X_{T}^{2}\right)+\sigma\right]
$$

Therefore,

$$
\lim _{n \rightarrow \infty} \mathbb{E}\left[\mid \frac{1}{n \delta_{n}} \int_{0}^{n \delta_{n}} X_{t}^{2} d t-\frac{1}{n} \sum_{k=0}^{n-1} X_{k \delta_{n}}^{2} \|\right]=0
$$

Let $\left.f: \mathbb{R}_{+}^{*} \times\right] 1 / 2,1\left[\times \mathbb{R}_{+} \rightarrow \mathbb{R}\right.$ be the continuous map defined by:

$$
f(u, v, w):=\frac{1}{1-\beta}\left[\frac{w}{v^{2}(1-\beta)^{2} u \Gamma(2 u)}\right]^{-1 /(2 u)}
$$

for every $\left.u \in \mathbb{R}_{+}^{*}, v \in\right] 1 / 2,1\left[\right.$ and $w \in \mathbb{R}_{+}$. By Theorem 5, Proposition 7, Proposition 8 and (5):

$$
\widehat{v}_{n}^{*}=f\left(\widehat{H}_{n}, \widehat{\sigma}_{n}, \frac{1}{n} \sum_{k=0}^{n-1} X_{k \delta_{n}}^{2}\right) \underset{n \rightarrow \infty}{\stackrel{\mathbb{P}}{\longrightarrow}} v .
$$

That achieves the proof.

\section{Copyrights}

Copyright for this article is retained by the author(s), with first publication rights granted to the journal.

This is an open-access article distributed under the terms and conditions of the Creative Commons Attribution license (http://creativecommons.org/licenses/by/3.0/). 